G. Bruno - F. Merletti - A. Biggeri - G. Bargero •

S. Ferrero - G. Pagano - P. Cavallo-Perin

\title{
Fibrinogen and AER are major independent predictors of 11-year cardiovascular mortality in type 2 diabetes: the Casale Monferrato Study
}

Received: 21 May 2004 / Accepted: 22 October 2004 / Published online: 5 February 2005

(C) Springer-Verlag 2005

\begin{abstract}
Aims/hypothesis: Fibrinogen and elevated AER increase cardiovascular mortality, but few data are available in the type 2 diabetic population. We have conducted an 11-year follow-up study of the Casale Monferrato cohort to assess: (1) the long-term predictive role of AER independently of conventional risk factors; (2) the shape of its relationship with cardiovascular mortality; and (3) whether fibrinogen has a predictive effect independent of the increased cardiovascular risk associated with nephropathy. Methods: During the follow-up period (1991-2001) a population-based cohort of 1,565 patients was regularly examined, and measurements of $\mathrm{HbA}_{1} \mathrm{c}$ were centralised. Multivariate Cox proportional hazards modelling was employed to assess the role of fibrinogen and AER as predictors of all-cause and cardiovascular mortality, independently of baseline variables and individual cumulative average values of $\mathrm{HbA}_{1} \mathrm{c}$ during follow-up. Results: In $10,890.2$ person-years of observations, 685 deaths were identified, giving an all-cause mortality rate of 63.4 per 1,000
\end{abstract}

G. Bruno $(\bowtie) \cdot S$. Ferrero $\cdot$ G. Pagano $\cdot$ P. Cavallo-Perin Department of Internal Medicine, University of Turin, corso Dogliotti 14,

10126 Turin, Italy

e-mail: graziella.bruno@katamail.com

Tel.: +39-011-6637434

Fax: +39-011-6634751

F. Merletti

Unit of Cancer Epidemiology, CERMS, University of Turin,

Turin, Italy

\author{
A. Biggeri \\ Department of Statistics "G. Parenti”, University of Florence, \\ Florence, Italy \\ G. Bargero \\ Santo Spirito Hospital, \\ Casale Monferrato, \\ Alessandria, Italy
}

person-years (95\% CI 58.8-68.3). In Cox regression analyses, the strongest predictor of cardiovascular mortality was macroalbuminuria (relative risk 2.18 , 95\% CI 1.62 2.94 ), which was mainly associated with a high risk of shortterm mortality. No increased risk was evident until the upper microalbuminuric range of AER values. Plasma fibrinogen was also a major independent predictor, and its role was not modified by AER, or by the exclusion of subjects developing chronic renal failure or diabetic nephropathy during followup. Conclusions/interpretation: The results indicate that: (1) AER is the main independent predictor of 11-year cardiovascular mortality; (2) this effect is mainly evident in the upper range of microalbuminuria and in macroalbuminuria; and (3) fibrinogen has an independent effect on cardiovasvascular mortality, but no synergistic effect with AER, suggesting that both endothelial dysfunction and chronic inflammation are involved in the excess cardiovascular mortality of type 2 diabetic patients.

Keywords Diabetic nephropathy $\cdot$ Fibrinogen $\cdot$ Mortality · Survey

Abbreviations HOPE: Heart Outcomes Prevention Evaluation - ICD: International Classification of Diseases · LR: Likelihood ratio chi square statistic $\cdot$ RR: Relative risk

\section{Introduction}

Albuminuria and fibrinogen are considered to be cardiovascular risk factors in both non-diabetic [1-4] and diabetic subjects [5-7]. Moreover, cross-sectional studies have provided evidence that fibrinogen levels are independently associated with AER in both type 1 and type 2 diabetic subjects [8-10]; however, the pathophysiological relationship between these two factors and the clinical relevance of their combined effect on cardiovascular risk remain unknown. Recent prospective data from the Casale Monferrato Study have provided evidence that hyperfibrin- 
ogenaemia independently predicts progression to overt diabetic nephropathy in type 2 diabetes [11]. Since cardiovascular mortality is higher in subjects with diabetic nephropathy than in subjects with diabetes alone [12-23], it is clinically relevant to assess whether the role of fibrinogen as a predictor of cardiovascular mortality is independent of baseline AER and progression to overt diabetic nephropathy. Albuminuria is considered to be a marker of endothelial cell damage, whereas fibrinogen is an indicator of chronic inflammation, which has been implicated in the pathogenesis of atherosclerosis and unstable atherosclerotic plaque in the coronary arteries. Therefore, the clinical usefulness of fibrinogen in the assessment of cardiovascular risk in diabetic patients would be increased if evidence were found that fibrinogen has a role that is independent of that of AER.

As regards albuminuria, its role as a cardiovascular risk factor in type 2 diabetic subjects has been highlighted in a systematic literature review [12] and in more recent studies [13-24]. However, the interpretation of the results from these studies has been hampered by: (1) the recruitment of clinic-based rather than population-based cohorts of subjects, which limits the extrapolation of the results to the whole diabetic population; (2) the small number of analysed events, which limits the power of the study for identifying risk factors and their interactions; and (3) the narrow spectrum of risk factors examined, which limits the assessment of inter-relationships between explanatory variables. Moreover, a number of critical questions have not been clarified as yet. Firstly, whether the shape of the relationship between AER and cardiovascular mortality is continuous or discrete. Only the Heart Outcomes Prevention Evaluation (HOPE) and the Prevention of Renal and Vascular End-Stage Disease (PREVEND) studies have previously examined this issue, reporting an increasing trend in cardiovascular risk even below the cut-off value for microalbuminuria, though this was assessed according to albumin: creatinine ratios and urinary albumin concentrations in these studies [13, 21]. Secondly, the role of AER as a predictor of long-term mortality in diabetic subjects has not been conclusively determined. Its role as a predictor of short-term mortality has only been suggested by studies based on few personyears of observations [24].

In this 11-year follow-up of the Casale Monferrato cohort of type 2 diabetic subjects we aimed to assess: (1) the longterm predictive role of AER, independently of conventional risk factors; (2) the shape of the relationship between AER and cardiovascular mortality; and (3) whether the predictive effect of fibrinogen is independent of the cardiovascular risk associated with nephropathy.

\section{Subjects and methods}

The study population consisted of 1,565 patients with known type 2 diabetes who were residents of the town of Casale Monferrato in the north-west of Italy $(93,477$ inhabitants) in 1988. Subjects were invited to attend a baseline examination in 1991-1992 to assess the prevalence of microalbuminuria, macroalbuminuria and cardiovascular risk factors, and were followed up through December 2001 $[25,26]$. They were identified using the following sources of ascertainment: (1) diabetes clinic; (2) general practitioners; (3) hospital discharges; (4) prescriptions; and (5) sale records of reagent strips and syringes. Patients cared for exclusively by their general practitioners comprised $23.8 \%$ of the cohort [27]. A high estimated completeness of ascertainment $(80 \%)$ was calculated by applying log-linear models to the capture-recapture methods, which take into account dependencies between data sources and heterogeneities of patients within sources [27]. Surveys conducted within Italy showed that the subjects were representative of Italian diabetic patients as regards age, sex, duration of diabetes, BMI and type of antidiabetic treatment.

As described in detail elsewhere, all patients were interviewed and examined by trained investigators at baseline after having provided informed consent [25]. The study was approved by the local ethics committee and was performed in accordance with the Declaration of Helsinki. Hypertension was defined as a systolic blood pressure $\geq 140 \mathrm{mmHg}$ and/or a diastolic blood pressure $\geq 90 \mathrm{mmHg}$ and/or treatment with antihypertensive drugs. Venous blood samples were collected in the fasting state for the determination of triglycerides, total cholesterol, HDL cholesterol (enzymatic-colorimetric method, after precipitation with $\mathrm{Mn}^{2+}$ ), $\mathrm{HbA}_{1} \mathrm{c}$ (Daiichi HPLC; Menarini, Florence, Italy; laboratory reference range $3.8-5.5 \%$ ) and fibrinogen (Clauss method) [10]. LDL cholesterol was calculated using Friedewald's formula. All laboratory determinations were centralised. AER was calculated on the basis of urinary albumin concentration measured in a single timed overnight urine collection by the nephelometric method (Behring Nephelometer Analyzer; Behring Institute, Marburg, Germany), after having excluded urinary tract infection, congestive heart failure or other known causes of non-diabetic renal diseases. Microalbuminuria and macroalbuminuria were defined as an AER 20-200 $\mu \mathrm{g} / \mathrm{min}$ and an AER $>200 \mu \mathrm{g} / \mathrm{min}$, respectively. Smoking habit was classified into one of three categories: never smoker, exsmoker (if the patient had stopped smoking at least 1 month prior to the visit) and smoker. For all patients enrolled, the date of diagnosis was retrieved and recorded. CHD was defined by ECG abnormalities according to the Minnesota code, as previously described [26].

During the follow-up period (1991-2001), patients were regularly examined (three to four times per year) either at the diabetes clinic or by general practitioners. As this prospective study was designed to determine the incidence of diabetic nephropathy and chronic renal failure [11, 28], $\mathrm{HbA}_{1} \mathrm{c}$, plasma creatinine and AER were regularly measured according to physician-scheduled plans at the main hospital in the area. This allowed us to retrospectively access a computerised database that included these centralised measurements. Individual average values for $\mathrm{HbA}_{1} \mathrm{c}$, which were updated at each follow-up examination, were calculated (cumulative individual averages).

The relevant time scale for the analysis was the time since diabetes diagnosis to death or to 31 December 2001. In- 
formation on deaths and the causes of death was obtained from the demographic files of towns of residence, hospital discharges and autopsy records. Only one patient was lost to follow-up. Underlying causes of death were derived and coded by two authors according to the ninth revision of the International Classification of Diseases (ICD). Diabetes was coded as the underlying cause of death in only 63 cases. Mortality rates were calculated by dividing the number of deaths that occurred during the study period by the number of person-years of observation.

Statistical analysis All continuous variables were categorised into quartiles of their distribution, apart from AER, which was categorised into normoalbuminuria, microalbuminuria and macroalbuminuria and into deciles, and age, which was categorised into 5-year age groups $(<60,60-64$, $65-69,70-74,75-79,>79)$. Analyses were performed using baseline variables. $\mathrm{HbA}_{1} \mathrm{c}$ values were measured at baseline and during follow-up, and were included in models using their cumulative individual average as a time-dependent variable. In all tables, age- and sex-adjusted relative risks (RRs) are shown. Estimations of $p$ values for trend were calculated using the Mantel-Haenszel method. The roles of AER and fibrinogen as predictors of cardiovascular and all-cause mortality, independently of conventional risk factors, were assessed with multivariate Cox proportional hazards models. Given the definition of the time scale, all models were also adjusted for known duration of diabetes. Variables included in the models fulfilled the proportional hazard assumptions, since the results of tests based on Schoenfeld residuals were not significant [29], apart from borderline significance $(p=0.06)$ for AER categories. The likelihood ratio test was used to assess the significance of variables. We tested for linear trend across categorical variables by entering a single ordinal term into the Cox regression model. In addition, we tested for deviation from linearity by comparing models containing AER values categorised into deciles with those containing a linear term in a likelihood ratio test with eight degrees of freedom.

In order to evaluate the combined effect of AER and fibrinogen on cardiovascular mortality, we divided the cohort into 12 groups according to normoalbuminuria, microalbuminuria and macroalbuminuria and quartiles of fibrinogen. In these analyses, Cox regression was used to simultaneously assess the risk of cardiovascular mortality in each of the 12 groups, with normoalbuminuria and the lowest quartile of fibrinogen as the reference group. The hypothesis that fibrinogen and AER have an interactive effect was tested in a Cox regression model that included a term for the multiplicative interaction of AER (across the three categories of normoalbuminuria, microalbuminuria and macroalbuminuria) and fibrinogen (across its quartiles). The $p$ value was two-sided, and a $p$ value of less than 0.05 was considered to indicate statistical significance. All analyses were performed using the STATA Statistical Software Package, Release 8.0 (STATA, College Station, TX, USA).

\section{Results}

At the baseline examination, the diabetic subjects had a mean $( \pm \mathrm{SD})$ age and diabetes duration of $68.9 \pm 10.7$ and $10.8 \pm 7.0$ years, respectively. As described previously [25], the prevalences of hypertension, microalbuminuria and macroalbuminuria were $84.5,31.9$ and $17.8 \%$, respectively. Patients cared for exclusively by their general practitioners comprised $23.7 \%$ of the cohort. Frequencies of treatment with diet, oral hypoglycaemic drugs and insulin were 12.2, 70.9 and $16.9 \%$, respectively.

During the 11-year follow-up period, 685 deaths were identified in 10,890.2 person-years of observations. This is equivalent to an all-cause mortality rate of 63.4 per 1,000 person-years (95\% CI 58.8-68.3). Cardiovascular mortality (ICD 390-459) accounted for 49.8\% (341/685) of deaths (mortality rate 31.5 per 1,000 person-years, $95 \%$ CI 28.4-35.1). Women had significantly lower age-adjusted mortality rates than men $(\mathrm{RR}=0.68,95 \%$ CI $0.58-0.80$ for all-cause mortality; and $\mathrm{RR}=0.75,95 \%$ CI $0.60-0.94$ for cardiovascular mortality).

Table 1 shows the age- and sex-adjusted relative risks for all-cause and cardiovascular mortality in the cohort. Increasing trends of risks were evident across AER categories and quartiles of fibrinogen and $\mathrm{HbA}_{1} \mathrm{c}$ cumulative average values. The risks were higher in smokers, in subjects with CHD, and in those cared for exclusively by their general practitioners. Cardiovascular risks were higher in patients with LDL : HDL cholesterol ratio values in the upper quartiles and in hypertensive subjects, though they did not reach statistical significance in the latter.

Predictors of cardiovascular and all-cause mortality In a multiple-risk-adjusted model including conventional cardiovascular risk factors (age, sex, hypertension, smoking and LDL : HDL cholesterol ratio) and $\mathrm{HbA}_{1} \mathrm{c}$ cumulative risk, microalbuminuria was the strongest independent predictor of cardiovascular mortality $(\mathrm{RR}=2.18,95 \%$ CI 1.62 2.94), whereas microalbuminuria was associated with a relative risk of 1.08 (95\% CI $0.82-1.45)$. A further adjustment for CHD only slightly modified the relative risks (1.14 in microalbuminuric subjects and 2.08 in macroalbuminuric subjects). Fibrinogen was also one of the main independent predictors of cardiovascular mortality: the relative risks were 1.41 (95\% CI 0.98-2.04), 1.55 (95\% CI $1.08-2.22), 1.63$ (95\% CI 1.15-2.31) in the lowest to the highest quartiles, respectively. Compared with patients cared for exclusively by general practitioners, patients cared for by diabetologists had a 29 and a 33\% lower risk of allcause and cardiovascular mortality, respectively, independently of other risk factors $(\mathrm{RR}=0.81,95 \%$ CI $0.67-0.98$; and $\mathrm{RR}=0.67,95 \%$ CI $0.51-0.87$, respectively). Further adjustment for antidiabetic treatment, antihypertensive treatments and triglycerides did not add significantly to the models.

When deciles of AER values were included in models, only high AER values were associated with increased risks (Table 2). A tendency towards a U-shaped curve, with large confidence intervals of point estimates, was evident, 
Table 1 All-cause and cardiovascular mortality rates in the population-based cohort of type 2 diabetes in the Casale Monferrato Study according to selected variables at baseline examination (1991-1992)

$\begin{array}{llll}\text { All-cause mortality } & \text { RR }^{\mathrm{a}}(95 \% \mathrm{CI}) & \begin{array}{l}\text { Cardiovascular mortality } \\ \text { Number of deaths }\end{array} & \begin{array}{l}\text { Rate per } 1,000 \\ \text { person-years }\end{array}\end{array} \quad \begin{aligned} & \text { Number of deaths } \\ & \text { Rate per 1,000 } \\ & \text { person-years }\end{aligned}$

\begin{tabular}{|c|c|c|c|c|c|c|}
\hline \multicolumn{7}{|l|}{$A E R$} \\
\hline Normoalbuminuria & 263 & 45.8 & 1.00 & 130 & 22.6 & 1.00 \\
\hline Microalbuminuria & 227 & 67.9 & $1.41(1.18-1.68)$ & 95 & 28.4 & $1.18(0.90-1.54)$ \\
\hline Macroalbuminuria & 168 & 118.0 & $2.29(1.88-2.79)$ & 99 & 69.2 & $2.73(2.10-3.57)$ \\
\hline$p$ value for trend ${ }^{\mathrm{b}}$ & & & $<0.0001$ & & & $<0.0001$ \\
\hline \multicolumn{7}{|l|}{ Fibrinogen $(g / l)$} \\
\hline$<3.00$ & 135 & 48.4 & 1.00 & 58 & 20.8 & 1.00 \\
\hline $3.00-3.49$ & 143 & 53.7 & $1.14(0.90-1.44)$ & 73 & 27.4 & $1.37(0.97-1.94)$ \\
\hline $3.50-4.10$ & 183 & 68.2 & $1.46(1.17-1.83)$ & 86 & 32.1 & $1.58(1.13-2.21)$ \\
\hline$>4.10$ & 191 & 76.1 & $1.48(1.18-1.85)$ & 101 & 40.2 & $1.77(1.28-2.46)$ \\
\hline$p$ value for trend $\mathrm{b}^{\mathrm{b}}$ & & & 0.0001 & & & 0.0002 \\
\hline \multicolumn{7}{|c|}{$H b A_{1} c$ cumulative average (\%) } \\
\hline$<6.4$ & 142 & 52.7 & 1.00 & 64 & 23.7 & 1.00 \\
\hline $6.4-7.7$ & 167 & 62.5 & $1.21(0.96-1.51)$ & 69 & 25.8 & $1.09(0.78-1.54)$ \\
\hline $7.8-9.6$ & 186 & 69.4 & $1.51(1.21-1.89)$ & 105 & 39.2 & $1.92(1.40-2.63)$ \\
\hline$>9.6$ & 155 & 60.1 & $1.49(1.18-1.88)$ & 78 & 30.2 & $1.66(1.18-2.33)$ \\
\hline$p$ value for trend ${ }^{\mathrm{b}}$ & & & $<0.0001$ & & & $<0.0001$ \\
\hline \multicolumn{7}{|c|}{ LDL : HDL cholesterol ratio } \\
\hline$<1.98$ & 175 & 66.9 & 1.00 & 70 & 26.8 & 1.00 \\
\hline $1.98-2.56$ & 143 & 54.9 & $0.84(0.67-1.05)$ & 76 & 29.1 & $1.12(0.81-1.56)$ \\
\hline $2.57-3.34$ & 163 & 61.8 & $1.03(0.83-1.28)$ & 84 & 31.8 & $1.37(1.00-1.88)$ \\
\hline$>3.34$ & 155 & 60.5 & $1.15(0.92-1.43)$ & 85 & 33.1 & $1.60(1.16-2.20)$ \\
\hline$p$ value for trend $\mathrm{b}^{\mathrm{b}}$ & & & 0.10 & & & 0.002 \\
\hline \multicolumn{7}{|l|}{ Hypertension } \\
\hline No & 91 & 52.9 & 1.00 & 37 & 21.5 & 1.00 \\
\hline Yes & 582 & 64.3 & $1.07(0.85-1.34)$ & 295 & 32.6 & $1.30(0.92-1.83)$ \\
\hline$p$ value & & & 0.47 & & & 0.09 \\
\hline \multicolumn{7}{|l|}{ Smoking } \\
\hline Never & 419 & 60.5 & 1.00 & 213 & 30.8 & 1.00 \\
\hline Ex & 147 & 69.1 & $1.40(1.10-1.77)$ & 71 & 33.4 & $1.49(1.06-2.10)$ \\
\hline Current & 87 & 57.4 & $1.52(1.16-1.98)$ & 38 & 25.6 & $1.49(1.01-2.20)$ \\
\hline$p$ value for trend $\mathrm{d}^{\mathrm{b}}$ & & & 0.002 & & & 0.052 \\
\hline \multicolumn{7}{|l|}{ CHD } \\
\hline No & 408 & 59.1 & 1.00 & 204 & 29.6 & 1.00 \\
\hline Yes & 168 & 79.4 & $1.25(1.04-1.50)$ & 19 & 42.1 & $1.33(1.03-1.71)$ \\
\hline$p$ value & & & 0.021 & & & 0.045 \\
\hline \multicolumn{7}{|l|}{ Physician } \\
\hline General practitioners & 180 & 79.5 & 1.00 & 101 & 44.6 & 1.00 \\
\hline Diabetologists & 502 & 59.3 & $0.87(0.73-1.04)$ & 238 & 28.1 & $0.76(0.60-0.96)$ \\
\hline$p$ value & & & 0.16 & & & 0.028 \\
\hline
\end{tabular}

${ }^{a}$ Adjusted for age and sex (given the definition of the time period, relative risks were also adjusted for duration of diabetes)

${ }^{\mathrm{b}}$ Based on the Mantel-Haenszel test for trend

with the lowest all-cause mortality risk in subjects with an AER between 11.68 and $15.00 \mu \mathrm{g} / \mathrm{min}$. Borderline significant deviations from linearity in relative risks for cardiovascular mortality were found by comparing nested models with decile indicators with those containing a linear term (likelihood ratio chi square statistic [LR], $[d f 8]=$ 15.90, $p=0.0438$ ) and by comparing a model with AER as a continuous variable with a model that also included a quadratic term $(\operatorname{LR}[d f 1]=3.76, p=0.0525)$.

The independent effect of macroalbuminuria on cardiovascular mortality diminished with length of attained time of follow-up ( $\mathrm{RR}=2.98,95 \%$ CI $0.95-9.41$ for length $<1.5$ years; and $\mathrm{RR}=1.53,95 \%$ CI $0.78-3.04$ for length $>6.0$ years). Indeed, 53 of the 168 (31\%) deaths that occurred 
Table 2 Relative risks of all-cause and cardiovascular mortality in the Casale Monferrato cohort of type 2 diabetes according to AER deciles

\begin{tabular}{lcc}
\hline $\begin{array}{l}\text { AER deciles } \\
(\mu \mathrm{g} / \mathrm{min})\end{array}$ & $\begin{array}{l}\text { All-cause } \\
\text { mortality } \\
\mathrm{RR}^{\mathrm{a}}(95 \% \mathrm{CI})\end{array}$ & $\begin{array}{l}\text { Cardiovascular } \\
\text { mortality } \\
\mathrm{RR}^{\mathrm{a}}(95 \% \mathrm{CI})\end{array}$ \\
\hline$<7.06$ & 1.00 & 1.00 \\
$7.06-9.09$ & $0.97(0.65-1.43)$ & $0.82(0.46-1.46)$ \\
$9.10-11.67$ & $0.91(0.61-1.37)$ & $0.89(0.51-1.55)$ \\
$11.68-15.00$ & $0.66(0.43-1.01)$ & $0.67(0.37-1.21)$ \\
$15.01-19.57$ & $1.02(0.69-1.51)$ & $0.87(0.50-1.51)$ \\
$19.58-26.66$ & $0.97(0.65-1.45)$ & $0.62(0.33-1.15)$ \\
$26.67-49.58$ & $1.26(0.86-1.85)$ & $1.07(0.62-1.83)$ \\
$49.59-143.28$ & $1.39(0.96-2.02)$ & $0.96(0.55-1.66)$ \\
$143.29-766.84$ & $1.81(1.25-2.62)$ & $1.67(0.99-2.79)$ \\
$>766.84$ & $1.71(1.18-2.48)$ & $1.78(1.06-2.97)$ \\
$p$ value for trend & $<0.001$ & $<0.001$ \\
\hline
\end{tabular}

${ }^{\mathrm{a}}$ Adjusted for age, sex, fibrinogen, $\mathrm{HbA}_{1} \mathrm{c}$ cumulative average, referring physician, LDL : HDL cholesterol ratio, hypertension and smoking

among macroalbuminuric patients occurred early (attained time of follow-up $<1.50$ years), and most of those who died did so within 6 years of follow-up $(145 / 168,86 \%)$. The corresponding values were 15 and $73 \%$ among microalbuminuric subjects, and 16 and $70 \%$ among normoalbuminuric subjects.

Effect of fibrinogen and AER Since subjects with chronic renal failure experience a higher rate of cardiovascular mortality than those without this complication, we performed analyses after excluding subjects who developed chronic renal failure $(n=72)$, defined as a creatinine value $>177 \mu \mathrm{mol} / \mathrm{l}$, during the 11-year follow-up period [28]. In this analysis, whereas the strength of other explanatory variables did not change markedly, the role of fibrinogen as an independent predictor became similar to that of macroalbuminuria $(\mathrm{RR}=1.84,95 \% \mathrm{CI} 1.23-2.76$ in the upper quartile of fibrinogen; and $\mathrm{RR}=1.80,95 \% \mathrm{CI} 1.26$ 2.58 in macroalbuminuric subjects). After further exclusion of subjects with CHD at baseline, the relative risk increased up to 1.97 in the upper quartile of fibrinogen
(95\% CI 1.16-3.35), and decreased to 1.75 (95\% CI $1.07-$ $2.85)$ in macroalbuminuric subjects.

The same analyses were repeated after excluding subjects who were macrolbuminuric at baseline $(n=268)$ and those who progressed to macroalbuminuria during the 6 years of follow-up $(n=202)$ [11]. In this analysis, the relative risk in the upper quartile of fibrinogen was $1.98(95 \%$ CI 1.13-3.37), whereas microalbuminuria was associated with a relative risk of 0.91 (95\% CI $0.61-1.37)$. When analyses were performed in the 371 normoalbuminuric subjects who remained normoalbuminuric at the sixth year of follow-up (30 deaths), the relative risks across quartiles of fibrinogen were (in ascending order): 1.22 (95\% CI $0.37-4.02), 2.42$ (95\% CI $0.74-7.93)$ and 0.91 (95\% CI $0.26-3.26)$ respectively.

In order to assess possible effect modification by fibrinogen on the AER predictivity of cardiovascular mortality, an analysis was performed in strata defined by the AER categories and fibrinogen quartiles combined. The results of Cox regression analyses (Table 3 ) show an independent effect of fibrinogen and AER categories on cardiovascular mortality, with the highest risk in macroalbuminuric subjects with fibrinogen levels $>3.00 \mathrm{~g} / \mathrm{l}$, but no evidence for an effect modification. Indeed, the expected relative ratios obtained by the multiplication of the relative ratios in each category of fibrinogen and AER are higher than the observed relative ratios. No significant interactions were found between AER categories and fibrinogen quartiles $(p=0.31)$.

\section{Discussion}

The results of the present analyses of the results of the population-based Casale Monferrato Study indicate that AER and fibrinogen are major predictors of cardiovascular mortality risk in type 2 diabetic subjects. These effects were independent of age, sex, duration of diabetes, blood pressure, lipids, smoking and glycaemic control during the study period, and were also independent of each other. No increased risk of cardiovascular mortality was evident within the normoalbuminuric range of AER values. The role of baseline fibrinogen as a predictor of cardiovascular mortality was not modified by AER, or by the exclusion of

Table 3 Modification effect of fibrinogen and AER on cardiovascular mortality in the Casale Monferrato cohort of type 2 diabetes

\begin{tabular}{|c|c|c|c|c|}
\hline & \multicolumn{4}{|l|}{$\mathrm{RR}^{\mathrm{a}}(95 \% \mathrm{CI})$} \\
\hline & Fibrinogen $<3.00 \mathrm{~g} / 1$ & Fibrinogen $3.00-3.49 \mathrm{~g} / 1$ & Fibrinogen $3.50-4.10 \mathrm{~g} / 1$ & Fibrinogen $>4.10 \mathrm{~g} / 1$ \\
\hline Normoalbuminuria & 1 & $1.40(0.76-2.58)$ & $2.07(1.17-3.66)$ & $1.69(0.93-3.08)$ \\
\hline \multirow[t]{2}{*}{ Microalbuminuria } & & $1.71^{\mathrm{b}}$ & $2.53^{\mathrm{b}}$ & $2.06^{\mathrm{b}}$ \\
\hline & $1.22(0.58-2.56)$ & $1.61(0.84-3.07)$ & $1.88(0.96-3.69)$ & $2.29(1.24-4.25)$ \\
\hline \multirow[t]{2}{*}{ Macroalbuminuria } & & $3.50^{\mathrm{b}}$ & $5.17^{\mathrm{b}}$ & $4.22^{\mathrm{b}}$ \\
\hline & $2.50(1.26-4.96)$ & $3.34(1.48-7.54)$ & $3.05(1.47-6.33)$ & $3.82(2.01-7.28)$ \\
\hline
\end{tabular}

\footnotetext{
${ }^{a}$ Adjusted for age, sex, $\mathrm{HbA}_{1} \mathrm{c}$ cumulative average, LDL : HDL cholesterol ratio, referring physician, hypertension, smoking and CHD
}

${ }^{b}$ Expected relative risks based on the assumption of a multiplicative model of effect modification 
subjects who developed chronic renal failure or macroalbuminuria during follow-up. These findings are original; no previous study has had the statistical power to assess the inter-relationships between AER, fibrinogen and cardiovascular mortality. Furthermore, they are consistent with the current hypothesis that endothelial dysfunction and chronic inflammation are both involved in the excess cardiovascular risk associated with type 2 diabetes [30]. It has been suggested that the hyperglycaemia-induced formation of reactive oxygen species in mitochondria and the generation of AGE might be the link between endothelial cell damage and damage to glomerular cells, leading to albuminuria [30]. Fibrinogen has been found to predict progression of nephropathy [8-10], probably as a marker of renal vascular damage; however, its role in diabetic subjects could be even more complex than that of an indicator of atherothrombotic disease. Indeed, emerging evidence suggests that chronic inflammation may represent a component of the metabolic syndrome, as it has been associated with insulin resistance and cardiovascular risk factors in diabetic subjects $[31,32]$.

Although fibrinogen has previously been reported to predict cardiovascular mortality in the general population [1-4], no data are available on its predictive role in the diabetic population, apart from a Swedish study that included 321 men with diabetes [5]. In a clinic-based cohort of 363 Danish diabetic subjects, an excess risk of all-cause mortality was reported to be associated with increased inflammation-sensitive plasma proteins (fibrinogen, $\alpha 1$ antitrypsin, haptoglobin, ceruloplasmin and orosomucoid) [6]. Previous reports from the Casale Monferrato Study have shown that hyperfibrinogenaemia is common in type 2 diabetes and is independently associated with higher AER values [10]. Moreover, baseline fibrinogen levels predict progression to macroalbuminuria, independently of blood pressure, $\mathrm{HbA}_{1} \mathrm{c}$ cumulative average levels and plasma lipids, suggesting that hyperfibrinogenaemia precedes overt diabetic nephropathy [11]. Whether or not the role of fibrinogen as a predictor of cardiovascular mortality is due to the increased cardiovascular risk associated with nephropathy has not previously been established. The present study is the first large population-based study on type 2 diabetes that has had the power to assess the predictive role of fibrinogen and its inter-relationships with classical risk factors and AER. Our results indicate that the predictive effect of fibrinogen is strong and independent of both classical risk factors and AER progression. Indeed, even after excluding patients who developed chronic renal failure or those who progressed to macroalbuminuria during followup, the predictive role of fibrinogen did not change. When analyses were performed in normoalbuminuric subjects who were still normoalbuminuric at the sixth year of followup, subjects in the second and the third quartiles of fibrinogen had higher relative risks than those in the lowest quartile, although the point estimates had large confidence intervals. The decrease in the point estimate of relative risk in the upper quartile is probably due to chance, as the analysis was based on only 30 deaths. Both AER and fibrinogen increased cardiovascular mortality, but we found no synergy between them in our analyses. Fibrinogen is considered to be an indicator of inflammation, which is now widely accepted as a contributory factor in the development of atherosclerosis. Alternatively, fibrinogen may be directly involved in atherosclerosis and thrombosis, since hyperfibrinogenaemia leads to enhanced coagulant activity and is associated with increased blood viscosity [33]. Our findings suggest that in diabetic subjects, this relatively inexpensive test may be useful in the identification of high-risk subjects. In diabetic subjects with plasma fibrinogen levels $>4.1 \mathrm{~g} / 1$ and macroalbuminuria, the risk of cardiovascular mortality is almost four times higher than that in normoalbuminuric subjects with plasma fibrinogen levels $<3.0 \mathrm{~g} / \mathrm{l}$. Lifestyle modifications [34] and drugs, such as bezafibrate [33], have been found to reduce fibrinogen levels, though not selectively, which prevents assessment of the effect of its reduction in intervention trials.

Endothelial dysfunction has been suggested as a link between increased AER and cardiovascular events in type 2 diabetes [30]. Our findings indicate that the association between AER and mortality is mainly evident in the upper range of microalbuminuria and in macroalbuminuria $(\mathrm{RR}=1.35$ and $\mathrm{RR}=2.04$ for all-cause mortality; and $R R=1.08$ and $R R=2.18$ for cardiovascular mortality, respectively), with no increased risk evident in normoalbuminuria. These results are at variance with the HOPE study, which found a continuously increasing risk in diabetic subjects even below the upper cut-off value for normoalbuminuria [13]. However, it should be noted that the Casale Monferrato cohort mainly included elderly subjects (representative of the Italian diabetic population) who were characterised by better glycaemic control and a later age at diagnosis than those recruited in studies performed in northern European countries and in the USA [26]. Furthermore, diabetic patients recruited in the HOPE study had at least one other cardiovascular risk factor, which increased the expected number of cardiovascular events during follow-up.

Although lower than previously reported from mainly clinic-based studies, our finding that microalbuminuria is associated with a $30 \%$ increase in all-cause mortality, even in the elderly, is relevant, highlighting the need for its primary prevention.

The Casale Monferrato Study recruited a prevalence cohort of patients at baseline (i.e. a heterogeneous cohort as regards duration of the disease and stage of its complications), and we found that an increase in the severity of the baseline risk factors (such as macroalbuminuria) was associated with an increase in short-term mortality. Indeed, among macroalbuminuric subjects, $31 \%$ of deaths occurred by the 1.5 -year follow-up and $86 \%$ of deaths occurred by the 6-year follow-up, compared with corresponding figures of 15 and $73 \%$ among microalbuminuric subjects and 16 and $70 \%$ among normoalbuminuric subjects.

The strengths of the present study are the prospective study design, the large population-based sample, and the long survival follow-up. The Casale Monferrato Study recruited a population-based cohort of subjects, allowing subjects cared for exclusively by general practitioners to 
be compared with those cared for by diabetes clinics. In a previous analysis of this cohort we found that patients with more severe diabetes were selectively referred to the clinics [26]. In spite of this, we found that patients cared for by the diabetic clinics had a 33\% lower risk of cardiovascular mortality. This finding is consistent with the results of a previous Italian study [35]. In Italy, all citizens are covered by the national health system; thus, the choice of care is not associated with socio-economic factors. However, our finding should be interpreted with caution, since it could be attributable to, among other factors, the different selfreferral patterns of patients.

In conclusion, this study demonstrates that, in type 2 diabetic subjects: (1) AER is the main independent predictor of 11-year mortality; (2) this effect is mainly evident in the upper range of microalbuminuric values and in macroalbuminuria; and (3) fibrinogen has an independent effect on cardiovascular mortality, but has no synergistic effect with AER. Therefore, both endothelial dysfunction and chronic inflammation are likely to be involved in the excess cardiovascular mortality in type 2 diabetic subjects.

Acknowledgements We thank patients, nurses of the diabetes clinic, diabetologists and general practitioners for their long-standing collaboration. The Casale Monferrato Study is supported by grants from the Ministry for University, Scientific and Technological Research (MURST), Italy. We also acknowledge the contribution of the Italian Association for Cancer Research (AIRC).

\section{References}

1. Tracy RP, Arnold AM, Ettinger W, Fried L, Meilahn E, Savage P (1999) The relationship of fibrinogen and factors VII and VIII to incident cardiovascular disease and death in the elderly: results from the cardiovascular health study. Arterioscler Thromb Vasc Biol 19:1776-1783

2. Yano K, Grove JS, Chen R, Rodriguez BL, Curb JD, Tracy RP (2001) Plasma fibrinogen as a predictor of total and causespecific mortality in elderly Japanese-American men. Arterioscler Thromb Vasc Biol 21:1065-1070

3. Yarnell JWG, Patterson CC, Sweetnam PM, Lowe GDO (2004) Haemostatic/inflammatory markers predict 10 -yr risk of IHD at least as well as lipids: the Caerphilly collaborative studies. Eur Heart J 25:1049-1056

4. Engström G, Hedblad B, Stavenow L et al (2004) Fatality of future coronary events is related to inflammation-sensitive plasma proteins. A population-based prospective cohort study. Circulation 110:27-31

5. Engström G, Stavenow L, Hedblad B et al (2003) Inflammation-sensitive plasma proteins, diabetes, and mortality and incidence of myocardial infarction and stroke. A populationbased study. Diabetes 52:442-447

6. Stehouwer CD, Gall M-A, Twisk JWR, Knudsen E, Emeis JJ, Parving H-H (2002) Increased urinary albumin excretion, endothelial dysfunction, and chronic low-grade inflammation in type 2 diabetes: progressive, interrelated, and independently associated with risk of death. Diabetes 51:1157-1165

7. Bosnyak Z, Forrest KY, Maser RE, Becker D, Orchard TJ (2003). Do plasminogen activator inhibitor (PAI-1) or tissue plasminogen activator PAI-1 complexes predict complications in type 1 diabetes: the Pittsburgh Epidemiology of Diabetes Complications Study. Diabet Med 20:147-151
8. Greaves M, Malia RG, Goodfellow K et al (1997) Fibrinogen and von Willebrand factor in IDDM: relationships to lipid vascular risk factors, blood pressure, glycaemic control and urinary albumin excretion rate: the EURODIAB IDDM Complications Study. Diabetologia 40:698-705

9. Klein RL, Hunter SJ, Jenkins AJ et al (2003) Fibrinogen is a marker for nephropathy and peripheral vascular disease in type 1 diabetes: studies of plasma fibrinogen and fibrinogen gene polymorphism in the DCCT/EDIC cohort. Diabetes Care 26:14391448

10. Bruno G, Cavallo-Perin P, Bargero G, Borra M, D'Errico N, Pagano G (1996) The associations of fibrinogen with glycemic control and albumin excretion rate in patients with non-insulindependent diabetes. Ann Intern Med 125:653-657

11. Bruno G, Merletti F, Biggeri A et al (2003) Progression to overt nephropathy in type 2 diabetes: the Casale Monferrato Study. Diabetes Care 26:2150-2155

12. Dinneen SF, Gerstein HC (1997) The association of microalbuminuria and mortality in non-insulin-dependent diabetes mellitus. A systematic overview of the literature. Arch Intern Med 157:1413-1418

13. Forsblom CM, Sane T, Groop P-H et al (1998) Risk factors for mortality in type II (non-insulin-dependent) diabetes: evidence for a role for neuropathy and a protective effect of HLA-DR4. Diabetologia 41:1253-1262

14. Gerstein HC, Mann JFE, Yi Q et al (2001) Albuminuria and risk of cardiovascular events, death, and heart failure in diabetic and nondiabetic individuals. JAMA 286:421-426

15. Valmadrid CT, Klein R, Moss SE, Klein BE (2000) The risk of cardiovascular disease mortality associated with microalbuminuria and gross proteinuria in persons with older-onset diabetes mellitus. Arch Intern Med 160:1093-1100

16. Yuyun MF, Dinnen SF, Edwards OM, Wood E, Warehan NJ (2003) Absolute level and rate of change of albuminuria over 1 year independently predict mortality and cardiovascular events in patients with diabetic nephropathy. Diabet Med 20:277-282

17. Chan JC, Cheung CK, Cheung MY, Swaminathan R, Critchley JA, Cockram CS (1995) Abnormal albuminuria as a predictor of mortality and renal impairment in Chinese patients with NIDDM. Diabetes Care 18:1013-1016

18. Agewall S, Wikstrand J, Ljungman S, Fagerberg B, Risk Factor Intervention Study Group (1997) Usefulness of microalbuminuria in predicting cardiovascular mortality in treated hypertensive men with and without diabetes mellitus. Am J Cardiol 80:164-169

19. Gall M-A, Borch-Johnsen K, Hougaard P, Nielsen FS, Parving $\mathrm{H}-\mathrm{H}$ (1995) Albuminuria and poor glycemic control predict mortality in NIDDM. Diabetes 44:1303-1309

20. MacLeod JM, Lutale J, Marshall SM (1995) Albumin excretion and vascular deaths in NIDDM. Diabetologia 38:610-616

21. Östgren CJ, Lindblad U, Melander A, Råstam L (2002) Survival in patients with type 2 diabetes in a Swedish community: Skaraborg Hypertension and Diabetes Project. Diabetes Care 25:1297-1302

22. Hillege HL, Fidler V, Diercks GFH et al (2002) Urinary albumin excretion predicts cardiovascular and non cardiovascular mortality in general population. Circulation 106:1777-1782

23. Fuller JH, Stevens LK, Wang S-L, WHO Multinational Study Group (2001) Risk factors for cardiovascular mortality and morbidity: the WHO multinational study of vascular disease in diabetes. Diabetologia 44(Suppl 2):S54-S64

24. Mattock MB, Barnes DJ, Viberti GC et al (1998) Microalbuminuria and coronary heart disease in NIDDM. An incidence study. Diabetes 47:1786-1792

25. Bruno G, Cavallo-Perin P, Bargero G et al (1996) Prevalence and risk factors for micro- and macroalbuminuria in an Italian population-based cohort of non-insulin-dependent diabetic subjects. Diabetes Care 19:43-47 
26. Bruno G, Cavallo-Perin P, Bargero G et al (1999) Cardiovascular risk profile of type 2 diabetic patients cared for by general practitioners or by a diabetes clinic: a population-based study. $\mathrm{J}$ Clin Epidemiol 52:413-417

27. Bruno G, LaPorte R, Merletti F, Biggeri A, McCarty D, Pagano G (1994) National diabetes programmes: application of capturerecapture to "count" diabetes? Diabetes Care 17:548-556

28. Bruno G, Biggeri A, Merletti F et al (2003) Low incidence of end-stage renal disease and chronic renal failure in type 2 diabetes. A 10-year prospective study. Diabetes Care 26:2353-2358

29. Schoenfeld D (1982) Partial residuals for the proportional hazards regression model. Biometrika 69:239-241

30. Ritz E (2003) Albuminuria and vascular damage. The vicious twins. N Engl J Med 348:2349-2352

31. Hanley AJG, Festa A, D'Agostino RB et al (2004) Metabolic and inflammation variable clusters and prediction of type 2 diabetes. Factor analysis using directly measured insulin sensitivity. Diabetes 53:1773-1781
32. Bruno G, Cavallo-Perin P, Bargero G et al (2001) Hyperfibrinogenemia and metabolic syndrome in type 2 diabetes: a population-based study. Diabetes Metab Res Rev 17:124-130

33. Ceriello A (1997) Fibrinogen and diabetes mellitus: is it time for intervention trials? Diabetologia 40:731-734

34. Kirk A, Mutrie N, MacIntyre P, Fisher M (2004) Effects of a 12-month physical activity counselling intervention on glycaemic control and on the status of cardiovascular risk factors in people with type 2 diabetes. Diabetologia 47:821-832

35. Zoppini G, Verlato G, Bonora E, Muggeo M (1999) Attending the diabetes center is associated with reduced cardiovascular mortality in type 2 diabetic patients: the Verona Diabetes Study. Diabetes Metab Res Rev 15:170-174 